\title{
TRANSFORMATIONS PRESERVING THE GRASSMANNIAN
}

\author{
BY \\ WILLIAM C. NEMITZ
}

1. Introduction. For $m$ a positive integer, let $E_{m}$ be the arithmetic $m$-space over a commutative field $F$. Let $\mathscr{A}_{m}$ be the full linear group of $E_{m}$, and let $S_{m-1}$ be the projective space of homogeneous coordinates in $E_{m}$. For the rest of the paper, we fix two positive integers $n$ and $k$, such that $k<n$. Let $N=\left(\begin{array}{l}n \\ k\end{array}\right)$, and let $\Omega(k, n)$ be the $k, n$ Grassmannian variety:

$$
\Omega(k, n) \subset S_{N-1} \text {. }
$$

Let $\psi(k, n)$ be the set of those nonzero elements $x$ of $E_{N}$ such that there is some $y$ satisfying

$$
x \in y \in \Omega(k, n) .
$$

Let $G$ be the set of nonsingular linear transformations of $E_{N}$ which keep $\psi(k, n)$ fixed as a set. If $C_{N}$ is the center of the full linear group of $E_{N}$, then $G / C_{N}$ is the set of projective transformations of $S_{N-1}$ which keep $\Omega(k, n)$ fixed as a set.

Let $A(n, k)$ be the group of all $k$-compounds [1, Vol. 1, p. 291] of elements of $\mathscr{A}_{n}$. Then $A(n, k) /\left(C_{N} \cap A(n, k)\right)$ may be thought of as the group of projective transformations of $S_{N-1}$ "induced" by the group of projective transformations of $S_{n-1}$. Since $A(n, k) /\left(C_{N} \cap A(n, k)\right)$ is isomorphic to $\left(A(n, k) \cdot C_{N}\right) / C_{N}$, and since $A(n, k) \cdot C_{N}$ is a subgroup of $G,\left(A(n, k) \cdot C_{N}\right) / C_{N}$ is a subgroup of $G / C_{N}$.

The principal results to be proved here are:

1. If $n \neq 2 k$, then

$$
A(n, k) \cdot C_{N}=G
$$

and thus

$$
\left(A(n, k) \cdot C_{N}\right) / C_{N}=G / C_{N} .
$$

2. If $n=2 k$, let $J$ denote the "star dual" mapping of $\psi(k, n)$ onto itself (see 2). Since

$$
J^{2}=(-1)^{\left(k^{2}\right)} I,
$$

where $I$ is the identity element of $\mathscr{A}_{N}, J$ generates a cyclic subgroup of order 2 if $k$ is even, and of order 4 if $k$ is odd. Let $\mathscr{J}$ denote this group. Let $\mathscr{K}$ be the

Received by the editors May 28, 1962 and, in revised form, December 12, 1962. 
subgroup of $G / C_{N}$ made up of cosets of elements of $\mathscr{J}$. Thus $\mathscr{K}$ is of order 2. Then, in this case,

and thus

$$
\mathscr{J} \cdot A(n, k) \cdot C_{N}=G
$$

$$
\mathscr{K} \cdot\left(\left(A(n, k) \cdot C_{N}\right) / C_{N}\right)=G / C_{N}
$$

2. Notation. (For definitions of terms used here and proofs of results given here, see [2].) We shall denote the exterior product of vectors by " $\wedge$ ". Thus $x$ is an element of $\psi(k, n)$ if and only if there is a linearly independent set of $k$ elements of $E_{n}, x_{1}, x_{2}, x_{3}, \cdots, x_{k}$; and

$$
x=x_{1} \wedge x_{2} \wedge x_{3} \wedge \cdots \wedge x_{k} .
$$

For $A \in \mathscr{A}_{n}$, let $A^{k}$ be the $k$-compound of $A$. Thus if

then

$$
x=x_{1} \wedge x_{2} \wedge x_{3} \wedge \cdots \wedge x_{k}
$$

$$
A^{k} x=A x_{1} \wedge A x_{2} \wedge A x_{3} \wedge \cdots \wedge A x_{k} .
$$

For $E \subset E_{m}$, let $L(E)$ be the subspace of $E_{m}$ spanned by $E$. If $x \in \psi(k, n)$, such that

let

$$
x=x_{1} \wedge x_{2} \wedge x_{3} \wedge \cdots \wedge x_{k}
$$

$$
\pi(x)=L\left(\left\{x_{1}, x_{2}, x_{3}, \cdots, x_{k}\right\}\right) .
$$

For any positive integer $m$, let

$$
\mathscr{N}(m)=\{1,2,3, \cdots, m\} .
$$

For $t$ a positive integer, $t \leqq m$, let

$$
\begin{gathered}
P(m, t)=\left\{p: p=\left\{p_{1}, p_{2}, p_{3}, \cdots, p_{t}\right\}, p_{i} \in \mathscr{N}(m) \text { for } i \in \mathscr{N}(t),\right. \text { and } \\
\left.p_{1}<p_{2}<p_{3}<\cdots<p_{t}\right\}
\end{gathered}
$$

For $p \in P(m, t)$, let $c(p)$ be that element of $P(m, m-t)$ such that

$$
p \cup c(p)=\mathscr{N}(m) \text {. }
$$

For $x$ an element of $\psi(k, n), * x$ is that element of $\psi(n-k, n)$ defined by

$$
(* x)_{q}=\varepsilon(q) x_{c(q)},
$$

where $q$ is any element of $P(n, n-k)$, and $\varepsilon(q)$ is -1 to the power of the parity of the permutation $\left(q_{1}, q_{2}, q_{3}, \cdots, q_{n-k},(c q)_{1},(c q)_{2},(c q)_{3}, \cdots,(c q)_{k}\right)$. Let $J$ be that mapping of $\psi(k, n)$ onto $\psi(n-k, n)$ defined by

$$
J(x)=* x
$$


Then $J$ can be extended to a nonsingular linear mapping of $E_{N}$ onto itself.

Since $k<n$, we may consider $E_{k+1}$ as a subspace of $E_{n}$, and $\psi(k, k+1)$ as a subset of $\psi(k, n)$. On occasion, we shall find it necessary to use the $*$-dual of a vector in $\psi(k, k+1)$ "relative to $E_{k+1}$." That is, for $x$ an element of $\psi(k, k+1) \subset \psi(k, n)$,

$$
\left(*_{k+1} x\right)_{i}=(-1)^{i-1} x_{c(i)}, \quad \text { where } c(i)=\mathscr{N}(k+1)-\{i\}, \text { if } 1 \leqq i \leqq k+1 ;
$$

and

$$
\left(*_{k+1} x\right)_{i}=0, \quad \text { if } i>k+1 \text {. }
$$

Then $*_{k+1} x \in E_{k+1} \subset E_{n}$, and

$$
L\left(*_{k+1} x\right)=(\pi(x))^{\perp_{k+1}},
$$

where $\perp_{k+1}$ denotes the orthogonal complement relative to $E_{k+1}$.

For $i \in \mathscr{N}(m)$, let $e_{i}$ be that element of $E_{m}$ whose $j$ th component is $\delta_{i j}$. For $p \in P(n, k)$, let

$$
e_{p}=e_{p_{1}} \wedge e_{p_{2}} \wedge e_{p_{3}} \wedge \cdots \wedge e_{p_{k}} .
$$

Then the set $\left\{e_{p}: p \in P(n, k)\right\}$ is a basis for $E_{N}$.

For $A \in G$, and $p \in P(n, k)$, let $A_{p}=A e_{p}$. Then $A_{p} \in E_{N}$, and it is the $p$ th column vector of the matrix of $A$. For any $q \in P(n, k-1)$,

$$
\operatorname{dim}\left(\bigcap \pi\left(e_{p}\right)\right)=k-1,
$$

the intersection being taken over all $p \in P(n, k)$ such that $q \subset p$; and

$$
\operatorname{dim}\left(L\left(\left\{e_{p}: q \subset p \in P(n, k)\right\}\right)\right)=n-k+1 .
$$

So if $A \in G$, and $q \in P(n, k-1)$, and if

$$
M=A\left(L\left(\left\{e_{p}: q \subset p \in P(n, k)\right\}\right)\right),
$$

then $\operatorname{dim} M=n-k+1$, and $M$ is spanned by the set $\left\{A_{p}: q \subset p \in P(n, k)\right\}$. Furthermore, for $p \in P(n, k), A_{p} \in M$ if and only if $q \subset p$.

Since we have excluded the zero vector from $\psi(k, n)$, no linear subspace of $E_{N}$ is contained in $\psi(k, n)$. However, if $M$ is a linear subspace of $E_{N}$, we shall say $M \subset \psi(k, n)$ if and only if for $x \in M$, if $x \neq 0$, then $x \in \psi(k, n)$.

3. Principal results. The principal results may now be stated in the following two theorems.

3.1. Theorem. If $n \neq 2 k$, and $A \in G$, then there exists $C \in C_{N}$ and $B \in \mathscr{A}_{m}$ such that

$$
A=C B^{k} \text {. }
$$

3.2. ThEOREM. If $n=2 k$, and if $A \in G$, then there exists $C \in C_{N}$ and $B \in \mathscr{A}_{n}$ such that either 


$$
A=C B^{k}
$$

or

$$
A=C J B^{k} \text {. }
$$

The proofs of these theorems depend on the following three lemmas, which will be proved in $\$ \S 4$ and 5 .

3.3. LeMmA. For $m$ an integer, $2 \leqq m \leqq N$, let $M$ be a subspace of $E_{N}$, with $\operatorname{dim} M=m$, such that there exists a set $\left\{x_{1}, x_{2}, x_{3}, \cdots, x_{m}\right\} \subset \psi(k, n)$ and $\left\{x_{1}, x_{2}, x_{3}, \cdots, x_{m}\right\}$ spans $M$. Then,

1. if

$$
\operatorname{dim} \bigcap_{i=1}^{m} \pi\left(x_{i}\right)=k-1,
$$

then $M \subset \psi(k, n)$,

and

$$
\operatorname{dim} \bigcap_{x \in M} \pi(x)=k-1,
$$

$$
\operatorname{dim} L(\{\pi(x): x \in M\})=k+m-1
$$

2. if

$$
\operatorname{dim} L\left(\left\{\pi\left(x_{i}\right): 1 \leqq i \leqq m\right\}\right)=k+1,
$$

then $M \subset \psi(k, n), m \leqq k+1$,

and

$$
\operatorname{dim} \bigcap_{x \in M} \pi(x)=k-m+1,
$$

$$
\operatorname{dim} L(\{\pi(x): x \in M\})=k+1 .
$$

In either case, $M$ is the set of all $k$-vectors of $k$ dimensional subspaces of $E_{n}$ which contain $\bigcap_{x \in M} \pi(x)$ and are contained in $L(\{\pi(x): x \in M\})$.

3.4. LEMMA. For $m$ an integer, $2 \leqq m \leqq N$, let $M$ be a subspace of $E_{N}$, with $\operatorname{dim} M=m$, and assume that $M \subset \psi(k, n)$. Let $\left\{x_{1}, x_{2}, x_{3}, \cdots, x_{m}\right\}$ be any spanning set of $M$. Then either

$$
\operatorname{dim} \bigcap_{i=1}^{m} \pi\left(x_{i}\right)=k-1,
$$

or

$$
\operatorname{dim} L\left(\left\{\pi\left(x_{i}\right): 1 \leqq i \leqq m\right\}\right)=k+1 .
$$

3.5. LEMMA. If $A \in G$, and if, for each $q \in P(n, k-1)$,

$$
\operatorname{dim} \bigcap \pi\left(A_{p}\right)=k-1,
$$

the intersection being taken over all p such that 


$$
q \subset p \in P(n, k),
$$

then there exists $C \in C_{N}$ and $B \in \mathscr{A}_{n}$ such that

$$
A=C B^{k} \text {. }
$$

Proof of Theorem 3.1 assuming Lemmas 3.3, 3.4, and 3.5. First assume that $n>2 k$. For $q \in P(n, k-1)$, let $M(q)$ be the subspace of $E_{N}$ spanned by the set $\left\{A_{p}: q \subset p \in P(n, k)\right\}$. Then $M(q) \subset \psi(k, n)$, and $\operatorname{dim} M(q)=n-k+1$. But $n-k+1>k+1$. So by 3.3 and 3.4 ,

$$
\operatorname{dim} \bigcap \pi\left(A_{p}\right)=k-1,
$$

the intersection being taken over all $p$ such that

$$
q \subset p \in P(n, k) \text {. }
$$

The result follows from 3.5. Now assume that $n<2 k$. Then for $x \in \psi(n-k, n), J A J^{-1}(x) \in \psi(n-k, n)$. Hence there exists $C \in C_{N}$ and $B \in \mathscr{A}_{n}$ such that

So

$$
J A J^{-1}=C B^{n-k}
$$

$$
A=C J^{-1} B^{n-k} J \text {. }
$$

By the Laplace expansion of a determinant,

$$
J^{-1} B^{n-k} J=(\operatorname{det} B) I\left(B^{-T}\right)^{k},
$$

where $-T$ denotes inverse transpose. Hence

$$
A=C(\operatorname{det} B) I\left(B^{-T}\right)^{k} .
$$

This completes the proof.

Proof of Theorem 3.2 assuming Lemmas 3.3, 3.4, and 3.5. We first show that if

$$
\operatorname{dim} L\left(\left\{\pi\left(A_{p}\right): q^{\prime} \subset p \in P(n, k)\right\}\right)=k+1,
$$

for some $q^{\prime} \in P(n, k-1)$, then

$$
\operatorname{dim} L\left(\left\{\pi\left(A_{p}\right): q \subset p \in P(n, k)\right\}\right)=k+1,
$$

for every $q \in P(n, k-1)$. It suffices to consider $q^{\prime}=\{1,2,3, \cdots, k-1\}$ and to assume that

$$
\operatorname{dim} L\left(\left\{\pi\left(A_{p}\right): q^{\prime} \subset p \in P(n, k)\right\}\right)=k+1 .
$$

Select $q \in P(n, k-1)$, so ordered that if $q_{i} \in q^{\prime}$, then $q_{i}=i$. Let $q^{\prime \prime}=\left\{2,3,4, \cdots, k-1, q_{1}\right\}$. We will show that

$$
\operatorname{dim} L\left(\left\{\pi\left(A_{p}\right): q^{\prime \prime} \subset p \in P(n, k)\right\}\right)=k+1 \text {. }
$$


If $q_{1}=1$, there is nothing to prove. So assume that $q_{1} \neq 1$. Let $p^{\prime \prime}=\left\{1,2,3, \cdots, k-1, q_{1}\right\}$, and let

and

$$
M^{\prime}=L\left(\left\{A_{p}: q^{\prime} \subset p \in P(n, k)\right\}\right),
$$

Then

$$
M^{\prime \prime}=L\left(\left\{A_{p}: q^{\prime \prime} \subset p \in P(n, k)\right\}\right) \text {. }
$$

so

$$
M^{\prime} \cap M^{\prime \prime}=L\left(A_{p^{\prime \prime}}\right)
$$

$$
\operatorname{dim}\left(M^{\prime} \cap M^{\prime \prime}\right)=1 .
$$

Now let $Q^{\prime}=L\left(\left\{\pi\left(A_{p}\right): q^{\prime} \subset p \in P(n, k)\right\}\right)$, and $Q^{\prime \prime}=\bigcap \pi\left(A_{p}\right)$, the intersection being taken over all $p \in P(n, k)$ such that $q^{\prime \prime} \subset p$, and assume that $\operatorname{dim} Q^{\prime \prime}=k-1$. Then

$$
Q^{\prime \prime} \subset \pi\left(A_{p^{\prime \prime}}\right) \subset Q^{\prime} \text {. }
$$

So the set of all $y \in \psi(k, n)$ such that $Q^{\prime \prime} \subset \pi(y) \subset Q^{\prime}$ is a subspace of $M^{\prime} \cap M^{\prime \prime}$, but by [1, Vol. 2, Chapter XIV, Theorem I], the dimension of this subspace is 2 . So $\operatorname{dim}\left(M^{\prime} \cap M^{\prime \prime}\right) \geqq 2$. This is a contradiction. So by Lemma 3.4 ,

$$
\operatorname{dim} L\left(\left\{\pi\left(A_{p}\right): q^{\prime \prime} \subset p \in P(n, k)\right\}\right)=k+1 .
$$

Continuing in this manner, working with one element of $q$ at a time, we conclude that

$$
\operatorname{dim} L\left(\left\{\pi\left(A_{p}\right): q \subset p \in P(n, k)\right\}\right)=k+1 .
$$

Hence either $A$ or $J A$ satisfies the conditions of Lemma 3.5, so the result follows from the fact that $J^{2}=(-1)^{\left(k^{2}\right)} I$.

4. Linear subspaces contained in $\psi(k, n)$. Lemmas 3.3 and 3.4 describe the linear subspaces of $E_{N}$ which are contained in $\psi(k, n)$ in the sense of 2 . In this section we give proofs of these two lemmas.

Proof of Lemma 3.3. Select a set $\left\{x_{1}, x_{2}, x_{3}, \cdots, x_{m}\right\} \subset \psi(k, n)$, such that $\left\{x_{1}, x_{2}, x_{3}, \cdots, x_{m}\right\}$ spans $M$, and assume that

$$
\operatorname{dim} \bigcap_{i=1}^{m} \pi\left(x_{i}\right)=k-1 .
$$

Then without loss of generality, we may assume that

$$
x_{i}=e_{1} \wedge e_{2} \wedge e_{3} \wedge \cdots \wedge e_{k-1} \wedge e_{k+i-1}, \quad \text { for } i=1,2,3, \cdots, m .
$$

Now let $x \in M$. Then there exist $a_{1}, a_{2}, a_{3}, \cdots, a_{m}$, elements of $F$, such that $x=\sum_{i=1}^{m} a_{i} x_{i}$. So

$$
x=e_{1} \wedge e_{2} \wedge e_{3} \wedge \cdots \wedge e_{k-1} \wedge\left(\sum_{i=1}^{m} a_{i} e_{k+i-1}\right)
$$


Hence $M \subset \psi(k, n)$, and consists of those $k$-vectors of $k$-spaces containing $L\left(\left\{e_{1}, e_{2}, e_{3}, \cdots, e_{k-1}\right\}\right)$, and contained in $L\left(\left\{e_{1}, e_{2}, e_{3}, \cdots, e_{k+m-1}\right\}\right)$. Now assume that

$$
\operatorname{dim} L\left(\left\{\pi\left(x_{i}\right): 1 \leqq i \leqq m\right\}\right)=k+1 .
$$

Then without loss of generality, we may assume that

$$
\pi\left(x_{i}\right) \subset L\left(\left\{e_{1}, e_{2}, e_{3}, \cdots, e_{k+1}\right\}\right)
$$

for $i=1,2,3, \cdots, m$. Hence the $x_{i}$ may be thought of as $k$-vectors in $E_{k+1}$. So if $x \in M, x=\sum_{i=1}^{m} a_{i} x_{i}$, for suitable elements $a_{i}$ of $F$, then $x$ is a $k$-vector in $E_{k+1}$. Hence $M \subset \psi(k, n)$, and

$$
\operatorname{dim} L(\{\pi(x): x \in M\})=k+1 .
$$

Also, the set $\left\{*_{k+1} x_{i}: 1 \leqq i \leqq m\right\}$ spans an $m$-space of $E_{k+1}$, so $m \leqq k+1$, and since $L\left(*_{k+1} x_{i}\right)=\left(\pi\left(x_{i}\right)\right)^{\perp_{k+1}}$,

$$
\operatorname{dim} \bigcap_{i=1}^{m} \pi\left(x_{i}\right)=k-m+1 .
$$

But for $x \in M, L\left(*_{k+1} x\right) \subset L\left(\left\{*_{k+1} x_{i}: 1 \leqq i \leqq m\right\}\right)$, and so

Hence

$$
\bigcap_{i=1}^{m} \pi\left(x_{i}\right) \subset \pi(x) .
$$

$$
\operatorname{dim} \bigcap \pi(x)=k-m+1,
$$

the intersection being taken over all $x \in M$. This completes the proof.

Proof of Lemma 3.4. Since $M \subset \psi(k, n)$, the plane spanned by $x_{i}$ and $x_{j}$ lies in $\psi(k, n)$, for $i \neq j, i, j=1,2,3, \cdots, m$. By [1, Vol. 2, Chapter XIV, Theorem I],

$$
\operatorname{dim}\left(\pi\left(x_{i}\right) \cap \pi\left(x_{j}\right)\right)=k-1 .
$$

So, without loss of generality, we may assume that

and

$$
\pi\left(x_{1}\right)=L\left(\left\{e_{1}, e_{2}, e_{3}, \cdots, e_{k}\right\}\right),
$$

$$
\pi\left(x_{2}\right)=L\left(\left\{e_{2}, e_{3}, e_{4}, \cdots, e_{k+1}\right\}\right) .
$$

Now assume that there is some $x_{j}$, say $x_{3}$, such that

$$
\pi\left(x_{1}\right) \cap \pi\left(x_{2}\right) \subset \pi\left(x_{3}\right) .
$$

Then we may assume that $\pi\left(x_{3}\right)=L\left(\left\{e_{2}, e_{3}, e_{4}, \cdots, e_{k}, e_{k+2}\right\}\right)$. Now assume that there is some $x_{i}$, such that $\pi\left(x_{i}\right)$ does not contain $\pi\left(x_{1}\right) \cap \pi\left(x_{2}\right)$. Since

$$
\operatorname{dim}\left(\pi\left(x_{i}\right) \cap \pi\left(x_{1}\right)\right)=\operatorname{dim}\left(\pi\left(x_{i}\right) \cap \pi\left(x_{2}\right)\right)=k-1,
$$


we can choose a spanning set $\left\{u_{1}, u_{2}, u_{3}, \cdots, u_{k}\right\}$ for $\pi\left(x_{i}\right)$ such that $u_{i} \in \pi\left(x_{1}\right)$, for $i=1,2,3, \cdots, k-1$, and $u_{k} \in \pi\left(x_{2}\right)$. Hence

and so

$$
\pi\left(x_{i}\right) \subset L\left(\left\{e_{1}, e_{2}, e_{3}, \cdots, e_{k+1}\right\}\right)
$$

$$
\operatorname{dim}\left(\pi\left(x_{i}\right) \cap \pi\left(x_{3}\right)\right)<k-1 .
$$

But this contradicts the fact that $\operatorname{dim}\left(\pi\left(x_{i}\right) \cap \pi\left(x_{3}\right)\right)=k-1$. So

$$
L\left(\left\{e_{2}, e_{3}, e_{4}, \cdots, e_{k}\right\}\right) \subset \pi\left(x_{i}\right),
$$

and hence

$$
\operatorname{dim} \bigcap_{i=1}^{m} \pi\left(x_{i}\right)=k-1 .
$$

Thus far, we have shown that if any three of the spaces $\pi\left(x_{1}\right), \pi\left(x_{2}\right), \pi\left(x_{3}\right), \cdots, \pi\left(x_{m}\right)$ intersect in a $k-1$ space, then they all intersect in a $k-1$ space. Now assume that no three of these spaces intersect in a $k-1$ space. Hence, for $i \neq 1,2, \pi\left(x_{i}\right)$ does not contain $\pi\left(x_{1}\right) \cap \pi\left(x_{2}\right)$. So, as before, $\pi\left(x_{i}\right) \subset L\left(\left\{e_{1}, e_{2}, e_{3}, \cdots, e_{k+1}\right\}\right)$, and so

$$
\operatorname{dim} L\left(\left\{\pi\left(x_{i}\right): 1 \leqq i \leqq m\right\}\right)=k+1 .
$$

5. Proof of Lemma 3.5. The proof is in two parts.

PART 1. We first prove that, given the assumptions of the lemma, there is a set $\left\{x_{1}, x_{2}, x_{3}, \cdots, x_{n}\right\} \subset E_{n}$, such that

$$
\pi\left(A_{p}\right)=L\left(\left\{x_{p_{1}}, x_{p_{2}}, x_{p_{3}}, \cdots, x_{p_{k}}\right\}\right)
$$

for any $p \in P(n, k)$. The proof is by induction on the number of vectors which can be found satisfying (1). First note that the assumption that for any $q \in P(n, k-1)$, the dimension of the intersection of the spaces $\pi\left(A_{p}\right)$ for $q \subset p \in P(n, k)$ is $k-1$, implies that to each $q \in P(n, k-1)$ there is assigned in a one-to-one manner, a $k-1$ space $S(q)$ of $E_{n}$, such that

$$
S(q)=\pi\left(A_{p}\right) \cap \pi\left(A_{r}\right),
$$

for any $p \in P(n, k)$, and $r \in P(n, k)$, such that $p \neq r$, and $q \subset p \cap r$. Obviously, there is a set $\left\{x_{1}, x_{2}, x_{3}, \cdots, x_{k}\right\} \subset E_{n}$ such that if $p=\{1,2,3, \cdots, k\}$, then (1) is true. So, assume that there exists a set $\left\{x_{1}, x_{2}, x_{3}, \cdots, x_{t}\right\} \subset E_{n}$, for some integer $t, k \leqq t \leqq n-1$, such that (1) holds for any $p \in P(t, k)$. Let $p=\{1,2,3, \cdots, k-1, t+1\}$. Then there exists an $x_{t+1} \in E_{n}$ such that (1) holds for this $p$. Let $q$ be an element of $P(t, k-1)$, so ordered that if $q_{s} \in p$, then $q_{s}=s$. Let $\bar{p}=q \cup\{t+1\}$. We wish to show that (1) holds for $\bar{p}$. We now define a family of elements of $P(n, k)$ as follows: 


$$
\begin{aligned}
p(0) & =p, \\
p(j) & =(p(j-1)-\{j\}) \cup\left\{q_{j}\right\},
\end{aligned}
$$

for $j=1,2,3, \cdots, k-1$. We will show by induction on $j$, that (1) holds for each $p(j)$. This will complete the induction on $t$, since $\bar{p}=p(k-1)$. Obviously, (1) is true if $j=0$. Assume that, for some $j, 0 \leqq j<k-1$, (1) holds for $p(j)$. If $q_{j+1}=j+1$, then (1) holds for $p(j+1)$. So assume that $q_{j+1} \notin p$. We also assume that $q_{j+1} \neq k$. Let

$$
\begin{aligned}
p^{\prime} & =(p(j+1)-\{t+1\}) \cup\{k\}, \\
r & =(p(j+1)-\{t+1\}) \cup\{j+1\}, \\
Z(i) & =L\left(\left\{x_{1}, x_{2}, x_{3}, \cdots, x_{i}\right\}\right),
\end{aligned}
$$

for $i$ equal $t$ or $t+1$. Then

$\pi\left(A_{p(j+1)}\right) \cap Z(t)=\pi\left(A_{p^{\prime}}\right) \cap \pi\left(A_{p(j+1)}\right)=S\left(p^{\prime} \cap p(j+1)\right)$

and

$$
=\pi\left(A_{p^{\prime}}\right) \cap \pi\left(A_{r}\right)=L\left(\left\{x_{p(j+1)_{1}}, x_{p(j+1)_{2}}, x_{p(j+1)_{3}}, \cdots, x_{p(j+1)_{k-1}}\right\}\right),
$$

$$
\pi\left(A_{p(j)}\right) \cap \pi\left(A_{p(j+1)}\right)=S(p(j) \cap p(j+1)) .
$$

Since $p^{\prime} \cap p(j+1) \neq p(j) \cap p(j+1)$,

$$
\operatorname{dim}\left(\pi\left(A_{p(j+1)}\right) \cap \pi\left(A_{p(j)}\right) \cap Z(t)\right)<k-1 .
$$

Also, since $Z(t+1)$ is spanned by $\pi\left(A_{p(j)}\right) \cup Z(t)$,

and hence

$$
\operatorname{dim}\left(\pi\left(A_{p(j+1)}\right) \cap Z(t+1)\right)=k,
$$

$$
\pi\left(A_{p(j+1)}\right) \subset Z(t+1) .
$$

Therefore, (1) holds for $p(j+1)$. If $q_{j+1}=k$, interchange $k$ and $j+1$ in the argument above. This completes the proof of Part 1.

Part 2. As a consequence of Part 1 , there is an $H \in \mathscr{A}_{n}$ such that $A H^{k}$ is diagonal. Hence we can assume that $A$ is diagonal.

$$
A=\operatorname{diag}\left(a_{p}\right), \quad \text { for } p \in P(n, k) .
$$

Now select any two integers $g$ and $h$, such that $1 \leqq g, h \leqq n$, and $g \neq h$. Let $q$ and $r$ be two elements of $P(n, k-1)$, neither of which contains $g$ or $h$. Let

$$
\begin{aligned}
p & =q \cup\{g\}, \\
p^{\prime} & =q \cup\{h\}, \\
\bar{p} & =r \cup\{g\},
\end{aligned}
$$

and 
We want to show that

$$
\bar{p}^{\prime}=r \cup\{h\} \text {. }
$$

$$
a_{p} a_{\bar{p}}=a_{p^{\prime}} a_{\bar{p}} .
$$

As in Part 1, we construct two families of elements of $P(n, k)$.

$$
\begin{aligned}
& p(0)=p, \\
& p(j)=\left(p(j-1)-\left\{q_{j}\right\}\right) \cup\left\{r_{j}\right\},
\end{aligned}
$$

for $j=1,2,3, \cdots, k-1$ and

$$
\begin{aligned}
& p^{\prime}(0)=p^{\prime}, \\
& p^{\prime}(j)=\left(p^{\prime}(j-1)-\left\{p_{j}^{\prime}\right\}\right) \cup\left\{r_{j}\right\},
\end{aligned}
$$

for $j=1,2,3, \cdots, k-1$. Here we regard the $p(j)$ and $p^{\prime}(j)$ as so ordered that $g$ or $h$ is always the last element. It suffices to prove that

$$
a_{p(j-1)} a_{p^{\prime}(j)}=a_{p(j)} a_{p^{\prime}(j-1)}
$$

for $j=1,2,3, \cdots, k-1$. Let $y=e_{p(j-1)}+e_{p(j)}+e_{p^{\prime}(j)}+e_{p^{\prime}(j-1)}$. Then $y \in \psi(k, n)$. Therefore $A y \in \psi(k, n)$. Thus $A y$ satisfies the Plucker identities, one of which may be written as (2), since only these four components of $A y$ are not zero. Now let

$$
b(g, h)=a_{p} / a_{p^{\prime}}
$$

Then $b(g, h)$ is independent of $q$, and for any three integers $g, h$, and $s$, $1 \leqq g, h, s \leqq n$,

$$
b(g, s)=b(g, h) b(h, s) .
$$

Therefore, for $r \in P(n, k)$, and $r^{\prime}=\{1,2,3, \cdots, k\}$,

$$
a_{r}=\prod_{i=1}^{k} b\left(p_{i}, i\right) a_{r^{\prime}}
$$

where $\Pi$ here indicates product. So if $B \in \mathscr{A}_{n}$

$$
B=\operatorname{diag}(b(1,1), b(2,1), b(3,1), \cdots, b(n, 1)),
$$

and

$$
\lambda=\left(\prod_{i=1}^{k} b(1, i)\right) a_{r^{\prime}}
$$

then

$$
A=\lambda I B^{k}
$$

This completes the proof of Lemma 3.5. 
6. The orthogonal group. In this section we let $F$ be the field of real numbers. For $m$ a positive integer, let $\cdot$ denote the usual inner product of $E_{m}$, and $|v|$ the usual norm. For $A \in \mathscr{A}_{m}$, let $A^{(i)}$ denote the $i$ th row vector of the matrix of $A$.

6.1. Lemma. For $m$ and $A$ as above, if there exists a set $T \subset E_{m}$ such that

1. $e_{i} \in T$ for all integers $i, 1 \leqq i \leqq m$,

2. $A^{(i)} \in T$ for all integers $i, 1 \leqq i \leqq m$,

3. for all $v \in T, A v \in T$, and $A^{-1} v \in T$,

4. for all $v \in T,|A v|=|v|$,

then $A$ is orthonormal.

Proof. Since, for $v \in T, A^{-1} v \in T$, we have that

$$
|v|=\left|A A^{-1}(v)\right|=\left|A^{-1}(v)\right| \text {. }
$$

Now let $x_{i}=A^{-1} e_{i}$ for any integer $i, 1 \leqq i \leqq m$. Then $\left|x_{i}\right|=1$, and $A x_{i}=e_{i}$. Hence $A^{(i)} \cdot x_{i}=1$, and thus $\left|A^{(i)}\right| \geqq 1$. But

So

$$
\left|A A^{(i)}\right|^{2}=\sum_{j=1}^{m}\left(A^{(j)} \cdot A^{(i)}\right)^{2}=A^{(i)} \cdot A^{(i)}
$$

$$
\sum_{j=1, j \neq i}^{m}\left(A^{(j)} \cdot A^{(i)}\right)^{2}=A^{(i)} \cdot A^{(i)}\left(1-A^{(i)} \cdot A^{(i)}\right) .
$$

Hence $\left|A^{(i)}\right| \leqq 1$. Thus, for any integers $i$ and $j, 1 \leqq i, j \leqq m, i \neq j,\left|A^{(i)}\right|=1$, and $A^{(i)} \cdot A^{(j)}=0$. Hence $A$ is orthonormal.

6.2. TheOREm. Let $A \in G$ such that for all $v \in \psi(k, n),|A v|=|v|$. Then $A$ is orthonormal, and there exist $B \in \mathscr{A}_{n}, B$ orthonormal, and $C \in C_{N}, C^{2}=I$, such that either $A=C B^{k}$, or $A=C J B^{k}$.

Proof. This follows immediately from the previous lemma.

\section{REFERENCES}

1. W. V. D. Hodge and D. Pedoe, Methods of algebraic geometry, The University Press, Cambridge, 1947-1952.

2. N. Bourbaki, Eléments de mathématique, II, Chapitre 3, Algebre multilinéaire, Hermann, Paris, 1958.

SOUTHWESTERN AT MEMPHIS, Memphis, TenNessee 